\title{
Reduction of spondylolisthesis
}

\author{
Setti S. Rengachary, M.D., and Raju Balabhandra, M.D. \\ Department of Neurosurgery, Spine Surgery Service, Wayne State University School of Medicine, \\ Detroit Medical Center, Detroit, Michigan
}

\begin{abstract}
In this article the authors discuss the syndrome of isthmic spondylolisthesis occurring at the lumbosacral junction in adults, providing a description of the clinical syndrome, altered biomechanics, and imaging characteristics. The authors pose arguments in favor of reduction and instrument-assisted fusion. Their surgical technique is detailed. They describe transsacral interbody fusion in which fibular allograft and in situ fixation are used, which they consider the second-best alternative technique when attempts at reduction fail.
\end{abstract}

KEY WORDS - biomechanics • isthmic spondylolisthesis - lumbosacral spine

Although spondylolisthesis as a clinical entity was described as early as 1782 by the Belgian obstetrician Herbiniaux, ${ }^{2}$ the syndrome has become well defined and appropriately classified only in the past 50 years. Introduction of spinal fusion technology and, more recently, instrumented stabilization and reduction methods have led to greater choices in treatment options. Concurrent with this is the emergence of controversies as to the optimum choice of treatment for a given patient. There are no established treatment standards, only guidelines and options, because no prospective randomized controlled trials have been published. Most of the reported series comprise retrospective reviews, originating from major spine centers, and are biased by the reporting surgeons' personal observations and experiences. Nevertheless, some general biomechanical principles in the causation, progression, and treatment of sagittal-plane imbalance specific to this disorder are being recognized, as well as accepted.

The focus of this article is relatively narrow. It pertains only to spondylolisthesis at the lumbosacral junction, specifically to the slippage of L-5 onto S-1. Isthmic spondylolisthesis occurring in adults older than age 18 years is the subject of the discussion. The emphasis is on highgrade (III and IV) spondylolisthesis. Low-grade (I and II) isthmic spondylolisthesis without lumbosacral kyphotic deformity, degenerative spondylolisthesis, congenital (dysplastic) spondylolisthesis, spondyloptosis, acute posttraumatic spondylolisthesis, and malignancy-induced spondylolisthesis are all excluded from this review for the

Abbreviation used in this paper: $\mathrm{VB}=$ vertebral body. following varying reasons: lack of controversy in management, rarity of the disorder (spondyloptosis), or occurrence only in pediatric patients.

\section{BIOMECHANICAL ALTERATIONS IN HIGH-GRADE LUMBOSACRAL ISTHMIC SPONDYLOLISTHESIS}

Once primates, including humans, evolved into beings with an upright posture a biomechanical strain occurred at the lumbosacral junction even under normal physiological conditions. The center of gravity falls anterior to the lumbosacral articulation. The lumbosacral disc space is normally slanted forward and downward. This leads to a shear force in the disc space and a rotational moment tending to drive the lower lumbar spine forward and to rotate in flexion. The anatomical feature that blocks these potentially harmful forces is the coronally oriented L5-S1 facet articulation. A lytic defect in the pars interarticularis allows these forces to act unchecked, resulting in progression of the slippage.

Over time, these forces create characteristic structural changes in the lumbosacral articulation that are readily recognizable on neuroimaging studies. There is an accelerated degeneration of the L5-S1 disc, resulting in desiccation, and a backward bulge of the anulus fibrosis occurs-the typical pseudodisc protrusion. The degeneration is generally distributed uniformly throughout the disc, with associated disc bulges at the anterior and posterior margins. In some instances, the intervertebral degenerative changes may extend to that above-namely, the L4-5 disc space - and may indeed be an additional source of pain. 
Characteristic changes occur in the endplates as well. Because of the slippage, the surface area of contact between the L-5 and S-1 endplates is reduced. Force concentration over a smaller area results in bone erosion in the posteroinferior corner of L-5 VB and the anterosuperior corner of the sacrum. The L-5 VB assumes a trapezoidal shape with a shorter posterior wall. The exiting L-5 nerve root, which wraps around the pedicle of L-5, tends to be directly over the L5-S1 interspace rather than behind the body of L-5 as it normally is. This altered anatomy poses the risk of injury to the L-5 nerve root as one attempts to perform a discectomy in preparation for an L5-S1 interbody fusion. The superior endplate of the sacrum becomes rounded and dome shaped because of erosion of the sharp anterosuperior corner of the sacrum. This typical morphological alteration results in forward pivoting of the L-5 VB around the sacral dome, causing progressive kyphotic deformity and, thus, an increase in angle of the slippage.

Progressive geometrical changes in the lumbosacral junction are manifest clinically by compensatory postural changes characteristic of high-grade spondylolisthesis. There is a compensatory hyperlordosis of the upper lumbar spine, a sustained postural contraction of the hamstrings in the upright position in an attempt to rotate the pelvis backwards, and because the trunk shortens with downward and forward protrusion of rib cage the buttocks appear flattened.

The most common neurological deficit is L-5 radiculopathy. The L-5 nerve root is entrapped - dorsally by a mass created by exuberant fibrous tissue at the isthmic defect and ventrally by an L5-S1 disc protrusion or the overriding posterosuperior corner of the sacrum. In rare cases the entire cauda equina roots may be compressed by the posterior dome of the sacrum, resulting in sphincter impairment.

\section{ARGUMENTS FOR REDUCTION SURGERY IN HIGH GRADE SPONDYLOLISTHESIS}

To correct or leave as is the high-grade slippage defect is the central controversial issue yet to be resolved in the surgical management of such high-grade lesions. In our judgment, this will cease to be an issue if all future cases are analyzed carefully and reduction surgery can be undertaken with a predictably low incidence of new neurological deficits. Again, it is our view that the exact mechanism of neurological damage during reduction surgery remains conjectural, although severe hypothetical mechanisms are proposed. Many are posited authoritatively but lack objective evidence to ground them. Future studies should be directed to performing sophisticated real-time continuous neural monitoring intraoperatively, objective intraoperative assessment of tension/stretch in the cauda equina nerve root, and any other test that will establish the cause of neural impairment.

For the purpose of this article, we will summarize the arguments for and against reduction surgery.

\section{Advantages of Reduction}

Spinal deformity may induce abnormal compensatory postural changes, slowly evolving neurological deficit from stretching or compression of neural elements, and chronic pain. One of the central goals of spine surgery is to correct spinal deformity to alleviate all these symptoms. In no other instance does a spinal surgeon consider not correcting an advanced symptomatic deformity. Why should high-grade spondylolisthesis be an exception?

The quality of fusion construct is optimized when the deformity is corrected. When reduction surgery is not performed, if lumbar interbody fusion (posterior or anterior) is considered, there is a smaller surface area of endplate cooptation for the placement of the graft. When the slippage is corrected, the surface area is restored. In cases in which conventional posterolateral fusion is considered, it is our observation that the transverse process of L-5 is small and hypoplastic. After removal of the L-5 posterior arch, including inferior articular facet, there is very little surface area left for a fusion bed. Additionally, without reduction, the graft is placed under tension if the transverse process continues its forward migration, a condition not conducive to fusion. This explains the low fusion rates with in situ arthrodesis. ${ }^{1}$

In situ fusions do not generally result in stable constructs. Even if osseous fusion is documented on x-ray film studies, the fusion mass may elongate under abnormal tensile forces and may result in progression of the vertebral slippage. Such a scenario is never known to occur in cases in which fusion is performed after reduction surgery.

In in situ fusion, the floating posterior arch of L-5 is generally not removed so as to provide a larger surface area for the fusion bed. Such a maneuver results in persistent and often worsening neurological deficit.

Spine surgeons who practice in situ fusion tend to include higher segments (namely L-4 and possibly L-3) to allow for a larger graft contact area. This practice is more likely to lead to junctional syndromes.

Because the sagittal deformity is not corrected by in situ fusion, compensatory postural changes tend to remain unchanged postoperatively. The patient's appearance and self-image are not helped.

Based on the aforementioned details, it is apparent that there are stronger arguments to correct such deformities if the surgical technique can be improved to minimize or avoid neurological deficits.

\section{REDUCTION TECHNIQUE}

The following description is the technique that we have used for reduction and stabilization. The paraspinal muscles are separated subperiosteally from the spinous processes and laminae of L-4, L-5, and sacrum. The L-5 spinous process is free floating because of lytic defect. Stabilization and fusion are performed to L-4 only when absolutely necessary. The L-5 posterior arch is completely removed either in one piece or in fragments, as is convenient. The L-5 posterior arch is typically very mobile. There is a forward slippage of the L-4 spinous process with respect to L-5. The posterior element of L-5 is divided into fragments after removal of soft tissues and saved. Although some authors have suggested keeping the posterior arch intact to serve a large surface area of fusion, its removal is necessary to achieve decompression of the L-5 
nerve root. It becomes a good source of autograft without the need to harvest iliac crest graft.

Additionally, removal of the L-5 arch allows direct visualization of the L-5 nerve root, which is at risk for injury during the reduction process. The entire intervertebral L5-S1 disc is removed piecemeal, excising with it as much as possible of the posterior longitudinal ligament. The goal is to disconnect the L-5 and S-1 vertebrae as completely as possible so as not to interfere with reduction. The cortical endplates of L-5 and S-1 are removed, resecting the dome of the sacrum.

Pedicle screws are now placed. The pedicle screw entry site for the sacrum is just inferior and lateral to its superior articular process. The first sacral pedicle is entered in a slow, incremental, fashion, using a "sacral sound" until the anterior cortex is just penetrated. Bicortical purchase certainly improves screw pullout strength. A trajectory is selected such that the tip is at the sacral promontory, the shaft of the screw being embedded in hard subchondral bone. The longest-diameter (usually 7.75 $\mathrm{mm}$ ) threaded screw affording good pullout strength is used. The length of the screw hole is precisely measured so as to allow the surgeon to choose the appropriate screw length. When the screw tip penetrates well into the pelvic cavity, pullout strength is not improved. Advancement of the screw tip toward the sacral promontory allows the spacing between L-5 and S-1 screw heads to prevent their knocking together.

The L-5 pedicle screw is placed next. The transverse process of L-5 and the superior articular process are rudimentary and deformed, and thus the usual anatomical landmarks for the pedicle screw entry site are not reliable; however, the inner surface of the L-5 pedicle is easily palpable with a ball-tipped Murphy probe. In fact the inner surface of the pedicle is quite often visible after the removal of the posterior arch. The deepest-length screw that can safely be placed within the anterior vertebral cortex is used. After tapping, the screws are placed bilaterally.

The reduction maneuver follows three steps: distraction, pulling back of the L-5 VB, and compression after graft placement in the intervertebral space.

The distraction is conducted by placing a long flat instrument (such as a shaver, a narrow Cobb elevator, or angled periosteal elevator) in the space and using it as a lever to distract the VBs. This distraction maneuver itself may allow partial correction of the forward slippage. Some surgeons have used Harrington distraction rods for this purpose, but we believe that intervertebral manipulation is simpler and requires less longitudinal soft-tissue dissection.

The longitudinal device connecting the screw tops can either be a rod or plate. We prefer to use the plate because it makes the reduction maneuver easier. The plate is contoured to accommodate the desired lumbar lordosis. The plate is anchored to the sacral screw first as the nut, anchoring the L-5 screw (usually a reduction screw with a long machine-threaded portion) until the plate is tightened. There is a pulling back of the L-5 VB toward the plate, which can be monitored on the fluoroscopy screen.

The intervertebral disc space is now packed using fusion material. Generally this includes a combination of local autograft, demineralized bone matrix, tricalcium phosphate granules, and tricortical allograft iliac crest. After the bone is firmly impacted, the securing nut over the L-5 pedicle screw is loosened, then retightened after compression. Generally Grade III or IV spondylolisthesis may be reduced to Grade I. Interbody arthrodesis is supplemented by posterolateral fusion. Using this technique, we have successfully performed this reduction surgery in seven consecutive cases of high-grade spondylolisthesis without neurological deficit.

In the event that screw placement in the L-5 pedicle is impossible because of anatomical constraints, then in situ fusion should be performed and followed by L4-S1 in situ fusion in high-grade spondylolisthesis is transsacral interbody fusion, ${ }^{3}$ using fibular allograft, generally $5 \mathrm{~cm}$ long. A sacral laminectomy is performed to the S-3 segment. The main dural sac is retracted laterally between the $\mathrm{S}-1$ and S-2 nerve roots to just beyond the midline. Using continuous live fluoroscopy a Steinmann pin is inserted to 3 to $5 \mathrm{~mm}$ short of the anterosuperior cortex of the L-5 VB. A cannulated anterior cruciate ligament reamer of incremental size is used until a 14-mm-diameter bone hole is reached. A fibular graft of precisely calculated length is cut and gently impacted into the drill hole until it approaches the anterior border of the L-5 VB. The fusion is supplemented by conventional intertransverse grafting and pedicle screw stabilization.

\section{CONCLUSIONS}

High-grade isthmic spondylolisthesis remains a challenging clinical problem, but surgery-related morbidity can be minimized when surgeons possess a good understanding of the altered anatomy, biomechanics, and principles of deformity correction and fusion.

\section{References}

1. Hanley EN Jr, Levy JA: Surgical treatment of isthmic lumbosacral spondylolisthesis. Analysis of variables influencing results. Spine 14:48-50, 1989

2. Neugebauer FI: The classic: a new contribution to the history and etiology of spondylolisthesis by F. L. Neugebauer. Clin Orthop 117:4-22, 1976

3. Smith JA, Deviren V, Berven S, et al: Clinical outcome of transsacral interbody fusion after partial reduction for high-grade L5-S1 spondylolisthesis. Spine 26:2227-2234, 2001

Manuscript received May 29, 2002.

Accepted in final form June 18, 2002.

Address reprint requests to: Setti S. Rengachary, M.D., Department of Neurosurgery, 4201 Street Antoine, Suite 6E, Detroit, Michigan 48201. email: srengachary@ neurosurgery.wayne.edu. 